\title{
LA REGULACIÓN EMOCIONAL COMO FACTOR TRANSDIAGNÓSTICO EN PSICOTERAPIA: \\ Luces y sombras de un campo en desarrollo
}

\section{EMOTION REGULATION AS A TRANSDIAGNOSTIC FACTOR IN PSYCHOTHERAPY: \\ Lights and Shadows of a Developing Field}

\author{
Fernández-Alvarez, J. ${ }^{1,2}$, Molinari, G. ${ }^{3}$, Colombo, D. ${ }^{2}$, García-Palacios, A. ${ }^{2,3}$, \\ Riva, G. ${ }^{1,4}$ y Botella, C.,3 \\ ${ }^{1}$ Università Cattolica del Sacro Cuore, Milán, Italia \\ ${ }^{2}$ Universitat Jaume I, Castellón, España \\ ${ }^{3}$ CIBER de Fisiopatología de la Obesidad y Nutrición, España \\ ${ }^{4}$ Applied Technology for Neuro-Psychology Lab, Istituto Auxologico Italiano, Milán, Italia
}

Cómo referenciar este artículo/How to reference this article:

Fernández-Alvarez, J., Molinari, G., Colombo, D., García-Palacios, A., Riva, G. y Botella, C. (2018). La regulación emocional como factor transdiagnóstico en psicoterapia: Luces y sombras de un campo en desarrollo. Revista de Psicoterapia, 29(110), 17-35.

\section{Resumen}

La regulación emocional se ha convertido a lo largo de las últimas dos décadas en uno de los constructos más estudiados en el campo de la psicología clínica. En el presente artículo se exponen en una primera sección los conceptos primordiales en torno a los hallazgos psicopatológicos que dan cuenta del papel primordial de la regulación emocional como factor transdiagnóstico. Posteriormente, se describen las nociones centrales que deben ser consideradas para llevar a cabo una mejor articulación entre investigación y práctica clínica. Por último, se presentan algunos de los principales tratamientos existentes que abordan la regulación emocional como eje central, para plantear en último lugar los caminos por los que el campo de la regulación emocional debería transitar como campo especifico de estudio en el contexto del desarrollo de la psicoterapia en general.

Palabras clave: regulación emocional, transdiagnóstico, integración en psicoterapia

\begin{abstract}
Over the last two decades, emotion regulation has become one of the most studied constructs in the field of clinical psychology. In the present article, we outline the main psychopathological findings that show the primordial role of emotion regulation as a transdiagnostic factor. Subsequently, the central notions that should be considered to move from a research dimension to an applied dimension are described. Finally, some of the main existing treatments that target emotion regulation as a nuclear axis are presented, to finally put forward the ways in which the field of emotion regulation should move as a specific field of study in the context of the development of the psychotherapy in general.

Keywords: emotion regulation, transdiagnostic, psychotherapy integration
\end{abstract}

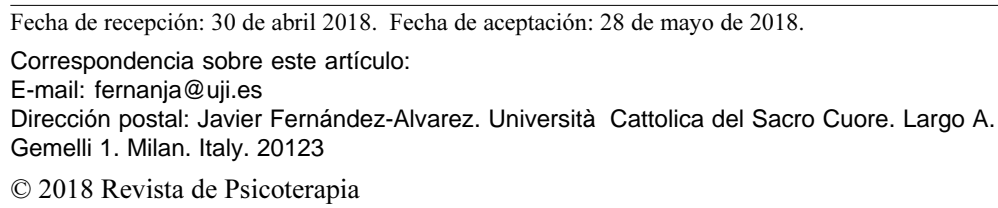


La regulación emocional se ha convertido a lo largo de las últimas dos décadas en uno de los constructos más estudiados en el campo de la psicología clínica. El crecimiento se ha manifestado de distintos modos, principalmente a través de la proliferación de las publicaciones sobre el tema, el particular interés que ha despertado en grupos con muy diversas perspectivas teóricas y metodológicas, así como su estudio en un amplio abanico de poblaciones sanas y con distintos niveles de disfunción clínica. Precisamente, el hecho de que grupos dedicados, tanto a la ciencia básica y experimental, como aquellos focalizados en el desarrollo de intervenciones y programas terapéuticos hayan puesto esfuerzos en operacionalizar y estudiar teórica y empíricamente el rol de la regulación emocional en condiciones muy diversas, ha hecho que se lo plantee como uno de los factores transdiagnósticos por excelencia.

En el presente artículo se exponen algunos de los principales desarrollos que se han elaborado en los últimos años respecto a la regulación emocional, con el objetivo de trazar los puntos fuertes y sobre todo las debilidades que deben afrontarse tanto en materia de investigación como en la práctica aplicada. Para ello, se plantea una primera parte en la que se exponen los conceptos primordiales en torno a los hallazgos psicopatológicos que dan cuenta del papel primordial de la regulación emocional como un factor transdiagnóstico. En segundo lugar, se describen las nociones centrales que deben considerarse para lograr articular investigación y práctica clínica. En una tercera parte, se describen algunos de los principales tratamientos existentes que abordan la regulación emocional como eje nuclear, planteándose finalmente los caminos por los que el campo de la regulación emocional debería transitar como campo específico de estudio en el contexto del desarrollo de la psicoterapia en general.

Conceptualización de la regulación emocional: factor transdiagnóstico en psicopatología

El principio básico que define la regulación emocional está asociado con el conjunto de procesos que las personas realizan individual o colectivamente, de un modo implícito o explícito, para maximizar la obtención de metas hedónicas o instrumentales. Comúnmente, la definición se enmarca en el modelo que desarrollara James Gross (1998) en el que la operacionalización del constructo se centra en la utilización de estrategias que las personas despliegan para lidiar con las emociones, tanto previa como posteriormente a su generación. Sin embargo, la perspectiva de estrategias ha sido complementada por una centrada en las habilidades que también ha visto un auge indudable durante la última década (Berking y Schwartz, 2014; Gratz y Roemer, 2004).

La naturaleza transdiagnóstica de la regulación emocional, se plantea a partir de la obtención de sólidos resultados empíricos que demuestran que ciertas estrategias de regulación emocional son factores desencadenantes y de mantenimiento de diversas condiciones clínicas (Aldao, Nolen-Hoeksema y Schweizer, 
2010; Webb, Miles y Sheeran, 2012). Habitualmente, se ha tendido a organizar dichas estrategias en una dicotomía esquemática, en función de los resultados (outcomes) derivados de su implementación; esto es, en estrategias adaptativas y desadaptativas. Por otra parte, a pesar de que se han desarrollado distintas taxonomías a partir de un gran número de estrategias diferentes, la mayor parte de los estudios se han centrado en la reevaluación cognitiva, la supresión, la rumiación o la aceptación.

Sin embargo, una cuestión preliminar que cabe preguntarse es si la regulación emocional constituye un proceso que puede apresarse conceptualmente de un modo claro y unánime. La definición de regulación emocional de James Gross ha sido habitualmente adoptada por la comunidad científica y, en rigor, está fundamentada en un modelo teórico sólido. De todos modos, no es menos cierto que la complejidad de la conducta humana hace difícil establecer cuándo el proceso de regulación es eminentemente emocional, cognitivo, psicofisiológico, comportamental o una compleja interacción entre dimensiones que resultan inseparables. La necesidad de circunscribir dicha complejidad bajo la etiqueta de regulación emocional ha llevado a que se genere un estiramiento conceptual tal que haya provocado que bajo su nombre se inscriban estudios y desarrollos heteróclitos que en algunos casos no son necesariamente compatibles.

El modelo de Gross constituye indudablemente el modelo más estudiado y por otra parte ha ido aportando reelaboraciones, por ejemplo la nueva perspectiva dinámica en la que se comprende mejor tanto el carácter procesual de la regulación emocional como la interacción entre generación y regulación emocional (Gross, 2015a). De todos modos, han aparecido otras propuestas al modelo de Gross, el cual ha sido criticado por estar fuertemente vinculado con un modelo cognitivo clásico que no contempla el carácter situado y encarnado del proceso regulatorio de las emociones (Koole y Veenstra, 2015). Por otra parte, el modelo de Gratz y Roemer (2004) y, en línea con este, el de Berking y Schwarz (2014) se han elaborado a partir del eje de las dificultades en la regulación de las emociones y las habilidades necesarias para revertir tales dificultades (es decir, centrado en la disfunción) por lo que se ha aplicado de manera más habitual en contextos de investigación clínica. Asimismo, otras de las puntualizaciones que se suele realizar al modelo de Gross es la focalización en una unidad de análisis individual, socavando el carácter interpersonal de la regulación de las emociones. En esa dirección existen diversos grupos trabajando tanto desde una perspectiva clínica como no clínica, dando cuenta de la relevancia de los procesos interaccionales (Dixon-Gordon, Bernecker y Christensen, 2015; Hofmann, 2014; Zaki y Williams, 2013). En conjunto, este corpus teórico y empírico pone de manifiesto la existencia de un campo sólido y en desarrollo que tiene en la integración de los distintos componentes el mayor desafío.

La posibilidad de incorporar una perspectiva integrativa reviste la necesidad de adoptar una concepción que abarque un modelo complejo de funcionamiento de la mente. Para ello es imprescindible captar que el acto fundamental que define a 
los seres humanos es el procesamiento de información con el objetivo de crear significados cuya complejidad y dinámica demanda una conceptualización conexionista que permita incorporar las más variadas situaciones que definen dicho proceso de creación y utilización de significados como puede ser la resolución de un problema de la vida cotidiana, afrontar situaciones dilemáticas o regular las emociones en situaciones difíciles. De este modo, los fenómenos clínicos y subclínicos se pueden comprender desde una concepción en la que la mente es: (a) dinámica para dar cuenta del carácter procesual que tiene la organización de la experiencia, (b) situada y encarnada pues la mente opera bajo determinadas condiciones como puede ser la configuración física y corporal y (c) extendida dado que la mente opera en contextos específicos en los que los mecanismos sociales, emocionales y cognitivos de regulación solo se pueden comprender en esa trama no lineal (Osbeck, 2009).

En este sentido, la concepción de regulación debe ser lo más amplia posible, entendiendo que un tratamiento clínico necesariamente debe apuntar a la desregulación para lograr una regulación adaptativa. El funcionamiento de todos los seres humanos, ya sea funcional o disfuncional, se enarbola sobre la base de un funcionamiento dialéctico en el que las personas se debaten entre modificar las necesidades contextuales y los cambios internos que consciente e inconscientemente buscan alcanzar, intentando preservar su unicidad como individuos a lo largo del tiempo y no perder de este modo la característica más esencial que define a las personas que es la identidad. Así, la regulación o desregulación emocional debe ser entendida como una conducta más que los individuos implementan con el objetivo de hacer frente a las demandas que la realidad les va imponiendo y por lo tanto un buen avance terapéutico supone la posibilidad de generar una organización de la experiencia más adaptativa para la persona que sufre. De este modo, una buena intervención terapéutica es aquella que toma en cuenta tanto las conductas disfuncionales que un individuo lleva a cabo como la experiencia en la que dicha conducta se materializa.

En esta dirección, el papel de la personalidad se vislumbra como un elemento de absoluta centralidad en el desarrollo de una concepción amplia e integrada, a partir del cual la disfunción y las características específicas puedan ser pensadas como una totalidad que se manifiesta en la inagotable diversidad de la que se compone la experiencia humana. Precisamente, en relación a la regulación emocional y la personalidad, se ha planteado recientemente una relación estrecha entre ambos constructos como factores predictivos psicopatológicos y sobre la necesidad de elaborar un modelo integrado que permita comprender la interacción entre ellos (Stanton, Rozek, Stasik-O'Brien, Ellickson-Larew y Watson, 2016). La evidencia comienza a mostrar que los rasgos de personalidad son tan predictivos como los rasgos clínicos (como el estilo rumiativo, la sensibilidad a la ansiedad, la supresión de pensamiento, etcétera) para el futuro desarrollo de, por ejemplo, los trastornos emocionales (Mahaffey, Watson, Clark y Kotov, 2016). 
Por otra parte, como complemento esencial para dicho esfuerzo teórico integrador en psicopatología, se requiere una reconfiguración profunda diagnóstica y de evaluación en general. El desarrollo del programa HiTOP (Kotov et al., 2017) es una buena referencia de que ya hay esfuerzos puestos en generar modelos alternativos que permitan reemplazar las vetustas y reificadas categorías diagnósticas por un sistema dimensional que redunde no solo en un mejor entendimiento de cómo se configuran las condiciones clínicas, sino sobre todo en mejores posibilidades de desarrollar programas efectivos de intervención terapéutica. De hecho, mientras no existían categorías definidas que compartimentalizarán la disfunción en etiquetas específicas, la concepción transdiagnóstica a partir de principios universales era en efecto más propensa a ser utilizada, tanto para la conceptualización psicopatológica, como para la intervención terapéutica (Sandín, Chorot y Valiente, 2012). Con el advenimiento de la denominada inflación diagnóstica, ha quedado de manifiesto que la utilidad de las clasificaciones diagnósticas categoriales y puramente sintomáticas constituyen un mapa muy distinto del territorio y que, por lo tanto, dificulta más que facilita la llegada a destino. En esta dirección, la perspectiva transdiagnóstica nació como un intento por conceptualizar la disfunción a partir del estudio de los procesos básicos como la atención, la emoción, el razonamiento o la memoria (Harvey, Watkins, Mansell y Shafran, 2004). En rigor, son dichos procesos que cuando resultan disfuncionales (e.g. sesgos atencionales, sobregeneralización de la memoria autobiográfica, sobreutilización de la rumiación sobre hechos pasados y del worry sobre eventos futuros) actúan como desencadenantes y mantenedores de la aparición de determinados síntomas que tradicionalmente han compuesto las categorías diagnósticas. Sin embargo, la dificultad de dejar de lado dichas categorías, por motivos que exceden el abordaje de este artículo, ha supuesto la proliferación transdiagnóstica desde una perspectiva centrada en los trastornos específicos. Esto es, en lugar de abordar el afecto negativo, la regulación emocional o los sesgos atencionales como algunos de los aspectos que comparten los trastornos emocionales, se ha tendido a seguir utilizando categorías como las de trastorno por pánico, trastorno de ansiedad generalizada, trastorno de ansiedad social, trastorno depresivo mayor, etcétera.

En ese sentido, la regulación emocional, entendida en su dimensión más amplia, como una parte más de la regulación general que cada individuo necesita construir, puede servir como punta de lanza para comprender que la verdadera concepción transdiagnóstica de la disfunción debe ser comprendida desde estos constructos supraordinales y no viceversa. En otras palabras, en lugar de conceptualizar que la regulación emocional y la regulación en su concepción más general está presente en varios trastornos y, por lo tanto, clasificarlo como transdiagnóstico, la necesidad de partir de la regulación como un aspecto que permite explicar la disfunción y acto seguido utilizarlo como un constructo necesario al margen de las categorías diagnósticas. Esta perspectiva está íntimamente relacionada con muchas otras conceptualizaciones dentro y fuera de la 
ciencia psicológica que dan cuenta de la necesidad de regulación como un mecanismo esencial en todo sistema. Desde el concepto de homeostasis en sistemas fisiológicos desarrollado por Walter Cannon sobre la base del trabajo hecho por Claude Bernard décadas antes (Cannon, 1932 ) o en el campo de la física y la ingeniería en el que incluso con anterioridad se hiciera énfasis en la necesidad de que un sistema mantuviese las propiedades a pesar de los cambios exógenos (Black, 1977), a las aplicaciones específicas en el campo de la psicología como las teorías de auto-regulación (Kanfer y Hagerman, 1987; Vohs y Baumeister, 2016), la concepción derivada de la teoría general de sistemas (Von Bertalanffy, 1967) o la teoría de control perceptual (Mansell y Marken, 2015).

Para ello, el desafío fundamental, además de las categorías diagnósticas, es desarrollar mejores estrategias de medición de la regulación emocional. Este es un desafío que excede a la regulación emocional y que supone generar mejores vías de aprehensión de los estados mentales, elemento constitutivo de la disciplina psicológica. Si no se piensa esto como un desafío fundamental, el advenimiento de las posturas reduccionistas - fisicalistas terminarán por relegar las posturas mentalistas, socavando a la psicología al campo neurocientífico. En particular, este debate ha cobrado fuerza a partir del nuevo marco de clasificación de trastornos mentales del National Institute of Mental Health, el Research Domain Criteria (RDoC), que contempla una serie de niveles para enfatizar la esencia dimensional de los trastornos mentales (Insel et al., 2010). La iniciativa RDoC ha suscitado fuertes debates respecto hasta qué punto considera los fenómenos psicológicos como un subproducto de un núcleo fundamental que estaría constituido por los procesos biológicos (Hershenberg y Goldfried, 2015).

Esto no quiere decir que disciplinas como la psicofisiología o la neurociencia no sean relevantes, sino refuerza la necesidad de integrar del modo más preciso posible las distintas perspectivas. De hecho, en la regulación emocional existen indicadores periféricos y neurales de la actividad fisiológica que constituyen elementos de suma utilidad para la investigación e incluso para la práctica clínica a través de técnicas como el biofeedback o el neurofeedback. En particular, la actividad parasimpática es aquella que se ha encontrado como el principal correlato de la respuesta emocional en general y de la regulación de las emociones en particular también. Las dos grandes teorías que apoyan este presupuesto son la Teoría Polivagal (Porges, 2007) y la Teoría de Integración Neurovisceral (Thayer y Lane, 2009). Dada las facilidades prácticas en su medición y la cantidad de evidencia que ha reunido en los últimos años, la variabilidad del ritmo cardíaco (HRV por sus siglas en inglés) y en especial la combinación del índice de la frecuencia alta con el ciclo respiratorio, que dan lugar a lo que se llama arritmia sinusal respiratoria (RSA por sus siglas en inglés), ha sido propuesto como un biomarcador de la regulación emocional (Beauchaine, 2015). Asimismo, las teorías anteriormente mencionadas, sobre todo la desarrollada por Thayer y Lane (2009), articulan el rol fundamental de la corteza prefrontal. Justamente la actividad central 
y el cerebro son indudables en el proceso de generación y regulación de las emociones, con estudios muy rigurosos tanto psicopatológicos como de intervención. Aunque un abordaje en detalle de esta cuestión merece un desarrollo mayor, cabe destacarse el rol central que juega la amígdala como responsable de las respuestas emocionales, la toma de decisiones y el procesamiento de la memoria. Sin embargo, hasta el momento no es posible determinar ni que la variabilidad del ritmo cardíaco ni los procesos cerebrales descritos, constituyan medidas de la regulación de las emociones sino meramente correlatos de la misma. En este sentido, a pesar de las dificultades de una evaluación de auto-informe, continúa siendo el mejor modo para conocer el estado emocional de una persona (LeDoux y Hofmann, 2017).

\section{La regulación emocional en psicoterapia}

La conexión entre psicopatología y psicoterapia en el campo de la regulación emocional puede leerse con un lente bifronte: por un lado, es indudable que muchos terapeutas utilizan principios, técnicas e intervenciones surgidos en el marco de la regulación emocional y, por lo tanto, es posible determinar que, a diferencia de otros constructos, existe una buena traslación entre ciencia y práctica. Sin embargo, hasta el momento el conocimiento en este campo es todavía limitado y por lo tanto los procedimientos psicoterapéuticos que existen no pueden escaparse de esa realidad. En rigor, lo que se suele desprender de una gran proporción de estudios clínicos es que determinadas estrategias de regulación emocional son adaptativas y otras desadaptativas, lo cual ni siquiera resulta fidedigno con la naturaleza compleja y contextual en la que se despliegan dichas estrategias. De allí es que el arsenal de herramientas terapéuticas pende de una máxima inespecífica: a mayor utilización de determinadas estrategias (sobre todo la reevaluación cognitiva y la aceptación), mejores resultados en salud mental y física, y a mayor utilización de otras estrategias (sobre todo, rumiación y supresión), menos salud mental y física. Cuando se intenta complejizar la ecuación, suele incluirse la noción de flexibilidad que supone el principio general de que lo realmente adaptativo es poder contar con un amplio menú de estrategias que es posible utilizar en diversos momentos según las necesidades personales y contextuales. De todos modos, el conocimiento a este respecto continúa siendo escaso y, sin duda, es necesaria mucha más investigación para poder decidir con precisión cuándo y qué tipo de estrategias de regulación emocional conviene recomendar a una determinada persona con un problema concreto, y luego cómo es posible desarrollar mecanismos precisos para promoverlas.

En ese sentido, y bajo las premisas generales que indican la necesidad de desarrollar una mayor flexibilidad, un mayor grado de estrategias adaptativas como la reevaluación cognitiva y aceptación, así como una disminución de estrategias desadaptativas como la supresión o la Rumiación, se han desarrollado diversos programas clínicos. Muchos de ellos son programas multitarget, es decir, que conceptualizan el abordaje de la regulación emocional como un componente más 
que se incluye junto con otros, como por ejemplo la activación conductual, la exposición, el entrenamiento en habilidades, etcétera. Por ejemplo, Gross (2015b) identifica 5 tipos de terapias en las que componentes de regulación emocional se han puesto a prueba con éxito: la Terapia de Regulación Emocional(Emotion Regulation Therapy), la Terapia Dialéctico-comportamental (Dialectical Behavior Therapy), la regulación de la emoción a través de la modificación de la atención (Regulation of Emotion through Modification of Attention), entrenamiento en regulación del afecto (basadas en estrategias) y las intervenciones en mindfulness.

\section{Terapia de Regulación Emocional (TRE) - Emotion Regulation Therapy (ERT)}

La TRE (Mennin y Fresco, 2009) es una intervención que incorpora los principios y hallazgos de la ciencia afectiva que apunta específicamente a intervenir en el distrés como factor transdiagnóstico entre distintas condiciones clínicas (Krueger y Markon, 2006), incluyendo el trastorno depresivo mayor y el trastorno de ansiedad generalizada. En consecuencia, la TRE trabaja sobre distintos mecanismos psicológicos sobre los que subyace el distrés, como la activación emocional motivacional o la perseveración en el pensamiento, con el objetivo de mejorar la regulación de las emociones. Se compone de 16 sesiones semanales, centrando inicialmente el foco en incrementar la conciencia motivacional (motivational awareness) y en desarrollar habilidades de regulación emocional. Después de esta primera parte psicoeducacional, se anima a los pacientes a practicar las nuevas habilidades a través de exposición experiencial con el fin de promover nuevos repertorios como consecuencia de someter a pruebas las habilidades en diversos contextos de aprendizaje.

\section{Terapia dialéctico-comportamental Dialectical Behavioral Therapy (DBT)}

La DBT se desarrolló en primer lugar para el trastorno límite de la personalidad (TLP), y tiene como objetivo mejorar las habilidades de las personas que padecen dicho trastorno, definido precisamente por la marcada tendencia a la inestabilidad emocional. El abordaje del TLP es lógicamente la condición clínica que reúne más evidencia en cuanto a volumen de estudios (Neacsiu, Bohus y Linehan, 2014); aunque no ha demostrado ser de mayor efectividad que otros abordajes (Cristea et al., 2017). Además, actualmente existen adaptaciones de la intervención para otras condiciones clínicas, como el trastorno bipolar (Guilleìn, Garciìa-Palacios y BanPos, 2018). La DBT, si bien también manualizada, tiene la enorme ventaja de ser una intervención integrativa que contempla diversos estadíos y una multiplicidad de dimensiones más allá de la reducción sintomática. Es particularmente útil la conceptualzación que realiza de la tensión entre identidad y cambio, aspecto que está en el centro de todas las condiciones con dificultad a la remisión, como es el caso de todos los trastornos de personalidad. 


\section{Modificación de la Atención - Modification of Attention (MA)}

Esta perspectiva se fundamenta sobre la base de que cuando se llevan a cabo estrategias desadaptativas, se producen sesgos en uno o más de los diferentes procesos cognitivos y psicológicos que todo individuo implementa. Un ejemplo lo constituye la atención selectiva, proceso que tiene un rol preponderante en la regulación de las emociones (Gross y Thompson, 2007) y, por lo tanto, los sesgos que se producen en su implementación han mostrado estar directamente asociados con pobres habilidades regulatorias (Mathews y MacLeod, 2005). En consecuencia, este abordaje apunta fundamentalmente a trabajar dichas respuestas selectivas, sobre todo a la información negativa, entrenando de este modo las habilidades atencionales (MacLeod y Grafton, 2014). Distintos estudios han mostrado resultados favorables de esta terapia, ayudando a los pacientes a mejorar las habilidades de regulación emocional y a la reducción sintomática (Dandeneau, Baldwin, Baccus, Sakellaropoulo y Pruessner, 2007; See, MacLeod y Bridle, 2009).

\section{Terapia de Regulación del Afecto (TRA) - Affect Regulation Training (ART)}

La TRA se basa en la integración de distintos abordajes terapéuticos como lo son la terapia cognitivo comportamental, la terapia dialéctico comportamental y las intervenciones a través del mindfulness. El principal aspecto que persigue radica en mejorar las habilidades de regulación emocional de los pacientes con distintas condiciones clínicas que comparten la desregulación de las emociones como característica transdiagnóstica (Berking et al., 2008; Berking, Ebert, Cuijpers, y Hofmann, 2013; Berking y Lukas, 2015). El modelo incluye un conjunto de habilidades que componen el núcleo del programa de intervención entre los que se promueve el reconocimiento y etiquetamiento de las emociones, la modificación activa de los estados afectivos, y la aceptación de emociones negativas, la aproximación y confrontación con estados afectivos negativos, la promoción de estados compasivos con uno mismo en situaciones estresantes, etcétera.

\section{Entrenamiento en mindfulness - Mindfulness Training (MT)}

Finalmente, otra intervención transdiagnóstica que apunta a la reducción del distrés y al mejoramiento de las habilidades de regulación de las emociones es el entrenamiento en mindfulness. Principalmente, pretende favorecer el desarrollo de la aceptación a través de atención centrada en el momento presente y constante que redunde en la posibilidad de experimentar conciencia plena (Williams, 2007). Las intervenciones en mindfulness pueden ser útiles para abordar tres aspectos de la regulación emocional (Farb, Anderson, Irving y Segal, 2014). En primer lugar, el incremento de la meta-conciencia sobre el proceso de regulación emocional, la facilitación de una mayor flexibilidad en dicha regulación y la adopción de estrategias de regulación con mayores niveles de conciencia en función de las necesidades personales y las demandas externas. En segundo lugar, el incremento de la atención respecto de los estados afectivos momentáneos contribuiría a la 
disminución de los procesos rumiativos, a una mayor detección de disparadores emocionales y a la elaboración de eventos negativos. Esta intervención se basa en la capacidad de desarrollar una atención focalizada través de prácticas meditativas y un monitoreo continuo de las sensaciones físicas como medio de acceso a la atención plena de pensamientos, emociones y experiencias.

A estos enfoques de tratamiento se podría agregar otros como el Protocolo Unificado de Barlow. De hecho, en España han aparecido distintas iniciativas que se han llevado a cabo desde la perspectiva transdiagnóstica a partir del Protocolo Unificado (Unified Protocol UP por sus siglas en inglés) de David Barlow, Allen y Choate (2004). Por un lado, en la Universidad Nacional de Educación a Distancia se está llevando a cabo a cabo un programa de validación y adaptación del UP a nivel grupal, del mismo modo que con esta perspectiva se han presentado datos preliminares en Argentina (Grill, Castañeiras, y Fasciglione, 2017). Asimismo, se está poniendo a prueba dicha perspectiva en distintas propuestas a nivel de atención primaria en España (Gonzailez-Blanch et al., 2018; Osma et al., 2018). Por último, de un modo innovador, se ha comenzado a aplicar este abordaje a través de intervenciones online, con dos ensayos clínicos controlados que ya están en marcha (Diìz-Garciìa et al., 2017; González-Robles et al., 2015).

Sin embargo, a pesar de la proliferación de intervenciones que se inscriben bajo la denominación de regulación emocional, no resultan claras y evidentes las diferencias que se pueden establecer entre los actuales repertorios de intervenciones basados en regulación emocional y la mayoría de las herramientas existentes en psicoterapia desde hace décadas. Por poner un ejemplo palmario, la reevaluación cognitiva constituye una de las estrategias por excelencia del programa que ofrece la regulación emocional para mejorar la condición clínica de las personas que poseen diversas condiciones clínicas. Sin embargo, ¿podría establecerse con claridad qué diferencia a las intervenciones en reevaluación cognitiva de aquellas que propusiera la terapia cognitiva a finales de los años 60 ? Si bien es cierto que se pone un énfasis mayor a la flexibilidad cognitiva en lugar de enfocarse necesariamente en los pensamientos negativos y las ideas irracionales, las diferencias parecieran ser más una discusión epistemológica y que inunda los ámbitos académicos que en diferencias sustanciales respecto a los procedimientos específicos que los terapeutas llevan a cabo.

Por su parte, los desarrollos y técnicas del movimiento denominado terapias de tercera generación o de tercera ola, recupera en mayor medida aspectos vinculados a la terapia de conducta clásica con herramientas que, o bien la terapia humanístico existencial había elaborado con anterioridad, o bien incorporando elementos de la tradición oriental, en particular de la filosofía budista (con el mindfulness a la cabeza), que también hacen dudar la novedad de los desarrollos que se plantean (Hofmann y Asmundson, 2008). En particular, el concepto de evitación experiencial puede estar revestido de componentes innovadores, pero en rigor, incluso semánticamente, recupera el elemento más constitutivo del modelo 
conductual que supone devolver el rol protagonista al principio de exposición. La ausencia de novedad no quita valor a las distintas propuestas, sino que enfatiza el carácter crecientemente integrativo que define a la psicoterapia, lo cual debe ser resaltado positivamente.

Por otro lado, los tratamientos descritos constituyen en su mayoría protocolos manualizados que han mostrado ser propicios para la puesta a prueba a través de ensayos controlados aleatorizados pero que luego esto mismo dificulta su adaptación a las necesidades específicas que se van planteando en la práctica clínica. Esto conlleva a una fuerte desarticulación entre lo que se investiga y lo que efectivamente luego se puede realizar en los consultorios. En el caso particular de los protocolos manualizados surge además un problema de inespecificidad cultural, dado que la mayoría de estos tratamientos se realizan y ponen a prueba en países desarrollados y las poblaciones en las que luego se aplican pueden resultar significativamente diferentes. Además, un problema adicional está constituido por las altas de comorbilidad que conllevan a que estos tratamientos que son diseñados y puestos a pruebo en su mayoría para trastornos específicos, y dado que en la práctica clínica raramente se encuentren pacientes con tanta pureza diagnóstica, se produce una nueva brecha entre lo que se investiga y lo que se practica.

Estas cuestiones no suponen críticas invalidantes a dichos protocolos como tampoco a la utilidad que pueda conllevar continuar con los programas de investigación tradicionales basados en el desarrollo de ensayos controlados aleatorizados. Sin embargo, en línea con lo que se viene planteando precisamente por representantes del desarrollo de la regulación emocional a través de modelos diversos como el de la TCC clásica y de los abordajes vinculados con la aceptación y el mindfulness, como lo son Stefan Hofmann y Steven Hayes respectivamente, han llamado a reducir la preponderancia de protocolos basados en la defensa acérrima de una escuela terapéutica o de una serie de técnicas que venga finalmente apoyado por evidencia de reusltados, para dar mayor énfasis a la evidencia de procesos y procedimientos. En una serie de nuevos artículos que han realizado de manera conjunta están promoviendo lo que han denominado terapia basada en procesos, en la que se pueda tender hacia la determinación de moderadores y mediadodres de cambio que permitan incrementar la personalización de los tratamientos ( Hofmann y Hayes, 2018).

Respecto a la regulación emocional en particular, la pregunta central gira en torno, entonces, a si la vasta evidencia existente para un conjunto amplio de condiciones clínicas convierte al constructo en verdaderamente relevante para la práctica psicoterapéutica. Si así lo fuese, ¿puede ser un indicador a tener en cuenta para saber quiénes se pueden beneficiar de un determinado tratamiento, o es un mecanismo que debe ser abordado para mejorar las diversas condiciones clínicas? Por otra parte, ¿en todas las condiciones clínicas las estrategias de regulación emocional se presentan del mismo modo?

En cuanto concierne la posibilidad de que la regulación emocional sea un 
constructo relevante para la psicoterapia, pueden mencionarse distintos ejemplos que ya han sido estudiados y que han arrojado evidencia preliminar al respecto. Por ejemplo, los niveles estables de reevaluación cognitiva o de rumiación que las personas utilizan, constituyen moderadores de la aparición subsecuente de síntomas depresivos (Shapero, Abramson y Alloy, 2016). De este modo, cabe pensar que las personas que reportan particulares dificultades en este tipo de estrategias, podrían ser buenos candidatos a la hora de llevar a cabo intervenciones preventivas. En la misma línea hay una vasta literatura que muestra el rol moderador de distintos componentes de la regulación de las emociones en la asociación entre factores de riesgo y resultados negativos (negative outcomes) como pueden ser el insomnio y la ansiedad (Kirwan, Pickett y Jarrett, 2017), la evitación emocional y también la sintomatología ansiosas (Bardeen, Tull, Stevens y Gratz, 2014), el estrés y el bienestar (Extremera y Rey, 2015) o los síntomas post-traumáuticos y el abuso de sustancias (Tull, Bardeen, DiLillo, Messman-Moore y Gratz, 2015), entre otros.

Por otra parte, la regulación emocional ha comenzado a reunir evidencia que indica la naturaleza mediadora en distintas situaciones, como en el apego inseguro (Beyderman y Young, 2016; Malik, Wells y Wittkowski, 2015), la adversidad acumulada a lo largo de la vida (Abravanel y Sinha, 2015) o situaciones traumáticas tempranas, y trastornos como la depresión y los trastornos de ansiedad y estrés. La identificación de mediadores se vincula con la posibilidad de determinar sobre qué aspectos específicos se debe intervenir para mejorar una condición clínica determinada. Sin embargo, resulta necesario tener en cuenta que para que sean sólidos los mediadores, deben poseer evidencia longitudinal y tal como marcan Sloan y colaboradores (2017) en un reciente artículo de revisión, en el campo de la regulación emocional la mayoría de la evidencia disponible es transversal. Además, la mediación se debe considerar para el establecimiento de un mecanismo como se considera a la correlación para el establecimiento de la causación: un elemento necesario pero no suficiente (Tyron, 2018). Y en este sentido todavía son escasos los desarrollos existentes.

Esta perspectiva que pretende tomar en consideración moderadores y mediadores es interesante como búsqueda de las variables que poseen peso en el desencadenamiento y mantenimiento de la conducta disfuncional. Asimismo, como se describía anteriormente, constituiría un modo de desarrollar intervenciones que se basen en una perspectiva de procesos, lo cual supondría una mejor articulación con la práctica clínica gracias a la posibilidad de considerar el carácter ideográfico con un mayor énfasis (Hofmann y Hayes, 2018).

Sin embargo, también cabe destacar que esta perspectiva supone la posibilidad de descomponer los fenómenos psicológicos en variables particulares, dejando de lado la indudable naturaleza emergente que implica la organización funcional o disfuncional de la experiencia. Por supuesto que no es un aspecto específico de la regulación emocional o del comportamiento disfuncional sino de la psicología en general, y aunque no debe desmerecerse la enorme utilidad de avanzar en esta línea 
de conocimiento, resulta indudable la necesidad de realizar un esfuerzo epistemológico por comprender cómo es posible la integración entre posiciones que tienden a focalizarse en los componentes específicos y aquellos que incorporan una noción compleja y por lo tanto no lineal de los fenómenos mentales sin perder por ello de vista la necesidad de obtener datos rigurosos y precisos. En este sentido resulta necesario darle un fuerte énfasis a la construcción teórica de manera integrativa, que permita conceptualizar los fenómenos desde una perspectiva amplia, y al mismo tiempo lograr evidencia empírica que permita apresar dicha complejidad. En esta dirección los desarrollos en minería de datos a través de estrategias de machine learning resultan una ventana interesante que si se logra articular con los datos clínicos reales puede ser preponderante para la personalización real de los tratamientos (Perna, Grassi, Caldirola y Nemeroff, 2018).

\section{Desarrollos para promover el avance del campo}

No puede dudarse entonces que existan protocolos que han mostrado ser eficaces para el tratamiento de diversos trastornos psicológicos, muchos de los cuales abordan específicamente la regulación de las emociones, tanto conceptual como técnicamente. Sin embargo, los tamaños del efecto de aquellas condiciones en las que la psicoterapia es más efectiva, como la depresión, siguen siendo pequeños (Cuijpers, Karyotaki, Reijnders y Ebert, 2018) y las tasas de prevalencia no disminuyen (Patten et al., 2016), con la salvedad que debe hacerse en las fobias específicas que sí han logrado desarrollar procedimientos efectivos con estabilidad de los resultados (Wolitzky-Taylor, Horowitz, Powers y Telch, 2008). Si bien es cierto que la diseminación es un componente fundamental dado que los terapeutas se basan más en la intuición y en la experiencia clínica que en los datos que arroja la investigación (Gyani, Shafran, Myles y Rose, 2014) es imprescindible detenerse en que esto ocurre como consecuencia del tipo de evidencia que se produce que, como se detallaba anteriormente, muchas veces resulta sumamente difícil de aplicar. Esto significa que simplemente intentando trasladar aquello que conocemos, no basta para afrontar un escenario en el que se avizora que los trastornos emocionales se conviertan para el año 2030 en la principal causa de incapacidad en el mundo, superando a las enfermedades cardiovasculares (WHO, 2017). En su lugar, se plantean dos caminos que de manera conectada pueden ser claves, ya sea para el caso de la regulación emocional en particular, como para el avance de la psicoterapia en general.

En primer lugar, es necesario reenfocar las preguntas centrales de investigación a partir de una promoción activa de colaboración entre clínicos e investigadores que salvo a través de un interés manifiesto y a contracorriente como ocurre en algunos casos, tiende a haber muy escasa articulación. El problema de la imposibilidad de generar un campo fértil en el que investigadores y clínicos dialoguen y construyan conocimiento de manera conjunta redunda en el socavamiento del fenómeno de interés común: ayudar a los pacientes a encontrar mejores modos de 
atravesar y resolver sus experiencias de sufrimiento. Si bien esto escapa al problema de la regulación emocional, se pone de manifiesto a través de distintas dimensiones en el plano específico como, por ejemplo, la utilización de instrumentos de evaluación que resultan poco útiles o las ya mencionadas estrategias esquemáticas que poco representan la complejidad de los problemas que presenten pacientes. En este sentido, si los investigadores y clínicos desarrollan proyectos colaborativos, no caben dudas de que el contenido generado más significativo y de mayor potencialidad de aplicabilidad. Las redes de colaboraciones de investigación-práctica que nacieron en Pennsylvania de la mano de Thomas Borkovec (2004) y que se diseminaron a lo largo y a lo ancho del mundo (Castonguay y Muran, 2015; Fernández-Álvarez, Fernández-Álvarez y Castonguay, 2018), resultan una promisoria vía para favorecer dicha colaboración.

Un segundo punto que resulta de sumo interés es la incorporación de la tecnología, en sus distintos desarrollos. Además de que hay muchos desarrollos propios que son potencialmente útiles para favorecer un marco de colaboración entre clínicos e investigadores (por ejemplo, las herramientas de monitoreo de pacientes a través de sistemas tecnológicos), existe un conjunto de avances específicos que prometen ser sumamente útiles, en principio para mejorar significativamente la investigación, pero también para la diseminación de aquellas intervenciones que muestren ser útiles para alguna condición clínica particular.

Por cuanto concierne a la investigación, los avances tecnológicos prometen ser sumamente beneficiosos desde el punto de vista experimental, por ejemplo a través de la utilización de eye tracker (Bebko, Franconeri, Ochsner y Chiao, 2011; Strauss, Ossenfort y Whearty, 2016) como también para el estudio observacional y clínico como pueden ser las intervenciones ecológicas momentáneas que permiten no solamente aprehender la variabilidad temporal sino además de un modo masivo gracias al alcance de los teléfonos móviles (Loo Gee, Griffiths y Gulliver, 2016; Miller, 2012).

Por su parte, para las intervenciones, como se mencionaba previamente, existen programas a través de internet que ya han mostrado ser útiles en la diseminación de los tratamientos existentes (Andersson, 2016). Hasta el momento constituyen fundamentalmente adaptaciones de las terapias tradicionales, pero se avizoran cambios que permitirán generar intervenciones customizadas a partir de las necesidades clínicas y de las preferencias del paciente. En particular, los abordajes a partir de internet pueden ser potenciados con la utilización de teléfonos móviles inteligentes, que no solo resultarán sumamente útiles en la intervención ecológica momentánea, sino sobre todo en la recolección de datos cognitivos, fisiológicos y comportamentales gracias a la incorporación de dispositivos portables con biosensores y evaluaciones momentáneas.

Por otro lado, la realidad virtual aparece como un desarrollo de enorme potencialidad, tanto para la investigación como para la práctica clínica. Desde el punto de vista de la investigación permite articular el campo experimental y 
aplicado en la medida de que los ambientes virtuales permiten el control necesario que se requiere para cumplir los criterios de manipulación experimental, pero en ambientes que pueden resultar significativos y reales. Esto último, precisamente, permite además que la investigación aplicada resulte útil para la práctica clínica dado que se basa en ambientes que pueden recrean los ámbitos naturales específicos de la vida cotidiana en los que las personas encuentran dificultades de distinto tipo. Es decir, permitiría una mayor personalización de las intervenciones (Botella, Baños, García-Palacios y Quero, 2017). Específicamente, para la regulación de las emociones, la realidad virtual permite la generación de ambientes en los que las personas deben desempeñarse de manera interactiva con el ambiente virtual a través de los que se pueden poner a prueba las estrategias específicas que la persona implementa ante determinadas situaciones. De este modo, es posible identificar no solo cómo la virtualidad incide sobre la persona, sino también el impacto que las respuestas emocionales, fisiológicas y conductuales de la persona tienen sobre el ambiente virtual, dando lugar así a un paradigma de interrealidad (interreality) (Gaggioli et al., 2014; Riva et al., 2010). Si bien hasta el momento el enfoque ha sido fundamentalmente para facilitar la exposición en el espectro de trastornos de ansiedad y del estrés, es posible avizorar un escenario en el que se desarrollen intervenciones específicas para entrenar al paciente en la modificación de una determinada estrategia desadaptativa o potenciar una adaptativa.

\section{Notas finales}

A lo largo de este artículo se han intentado trazar las luces y sombras del campo de la psicoterapia, en particular de los desarrollos en regulación emocional como constructo transdiagnóstico. Para ello, se ha puesto el foco en el péndulo que va de la investigación a la clínica, y viceversa, enfatizando los caminos transitados y los desafíos restantes para potenciar las intervenciones y lograr entonces ayudar a los pacientes a mejorar su calidad de vida.

Luego de haber repasado algunos de los principales aportes, entonces, ¿qué papel debe jugar la regulación emocional en el desarrollo de la psicoterapia? Desde una mirada acotada, en principio es posible avizorar un futuro en el que la regulación emocional tendrá un papel importante en la investigación y práctica clínica. La hipótesis de mínima, ya relativamente probada, es que sea un proceso relevante que en rigor se identifique en diversas condiciones clínicas y que, en todas o algunas de ellas, constituya un indicador de patología o de recuperación clínica. La hipótesis de máxima sería el desarrollo de un conjunto de técnicas específicas que permitieran identificar de qué modo se debe intervenir en la regulación emocional para mejorar distintas condiciones clínicas.

Sin embargo, una propuesta más revulsiva implica la necesidad de construir un modelo psicopatológico y como consecuencia de intervención verdaderamente transdiagnóstico e integrativo, en el que la regulación emocional se inserte como una de las tantas aristas a tener en cuenta. Esto es, un modelo psicopatológico que 
explique la disfunción más allá de los trastornos específicos. En este sentido, el ejercicio es doble: descentrar a la regulación emocional como único proceso relevante y desde la propia conceptualización teórica elaborar un modelo de regulación complejo. Por supuesto que esto atenta contra una posición, a veces dominante, de construir modelos explicativos a partir de la mera acumulación de datos empíricos. Por otro lado, el ejercicio consiste en pensar que ciertos procesos, como la regulación emocional, ya constituyen elementos relevantes en todas las conceptualizaciones teóricas derivadas de las distintas escuelas de pensamiento en psicología clínica.

En definitiva, la regulación como fenómeno transdiagnóstico, debe ser comprendida en el marco general del desarrollo en psicoterapia. Es decir, un terreno que con aspectos esperanzadores y otros menos, avanza hacia un desarrollo teórico integrado con un conjunto de intervenciones específicas que buscan insertarse en dicha conceptualización general. La regulación emocional y las distintas herramientas que facilitan el mejoramiento en su implementación serán elementos constitutivos de los tratamientos clínicos futuros, pero no debe correrse el riesgo de intentar situarla como la panacea de la psicología clínica.

\section{Agradecimientos}

Este trabajo fue apoyado por Marie $\mathrm{Sk}^{3}$ odowska-Curie Innovative Training Network AffecTech (ID del proyecto: 722022) financiado por la Comisión Europea H2020.

\section{Referencias bibliográficas}

Abravanel, B. T. y Sinha, R. (2015). Emotion dysregulation mediates the relationship between lifetime cumulative adversity and depressive symptomatology. Journal of Psychiatric Research, 61, 89-96. doi:10.1016/ j.jpsychires.2014.11.012

Aldao, A., Nolen-Hoeksema, S. y Schweizer, S. (2010). Emotion-regulation strategies across psychopathology: A meta-analytic review. Clinical Psychology Review, 30(2), 217-237. doi:10.1016/j.cpr.2009.11.004

Andersson, G. (2016). Internet-Delivered Psychological Treatments. Annual Review of Clinical Psychology, 12, 157-179. doi:10.1146/annurev-clinpsy-021815-093006

Bardeen, J. R., Tull, M. T., Stevens, E. N. y Gratz, K. L. (2014). Exploring the relationship between positive and negative emotional avoidance and anxiety symptom severity: the moderating role of attentional control. Journal of Behavior Therapy and Experimental Psychiatry, 45(3), 415-420. doi:10.1016/j.jbtep.2014.04.006

Barlow, D., Allen, L. B. y Choate, M. L. (2004). Toward a Unified Treatment for Emotional Disorders. Behavior Therapy, 35, 205-230.

Beauchaine, T. P. (2015). Respiratory sinus arrhythmia: a transdiagnostic biomarker of emotion dysregulation and psychopathology. Current Opinion in Psychology, 3, 43. doi:10.1016/j.copsyc.2015.01.017

Bebko, G. M., Franconeri, S. L., Ochsner, K. N. y Chiao, J. Y. (2011). Look before you regulate: differential perceptual strategies underlying expressive suppression and cognitive reappraisal. Emotion, 11(4), 732742. doi:10.1037/a0024009

Berking, M. y Schwartz, J. (2014). Affect regulation training. En J. J. Gross (ed.), Handbook of emotion regulation (2 ed., pp. 529 - 547). Nueva York, NY: Guilford Press. 
Beyderman, I. y Young, M. A. (2016). Rumination and overgeneral autobiographical memory as mediators of the relationship between attachment and depression. Personality and Individual Differences, 98, 37-41. doi:10.1016/j.paid.2016.03.077

Black, H. S. (1977). Inventing the negative feedback amplifier: Six years of persistent search helped the author conceive the idea "in a flash" aboard the old Lackawanna Ferry. Spectrum, IEEE, 14, 55-60. doi:dx.doi.org/ 10.1109/MSPEC.1977.6501721

Borkovec, T. D. (2004). Research in Training Clinics and Practice Research Networks: A Route to the Integration of Science and Practice. Clinical Psychology: Science and Practice, 11(2), 196-210. doi:10.1093/clipsy/ bph073

Botella, C., Baños, R. M., García-Palacios, A. y Quero, S. (2017). Chapter 22 - Virtual Reality and Other Realities A2 - Hofmann, Stefan G. En G. J. G. Asmundson (Ed.), The Science of Cognitive Behavioral Therapy (pp. 551-590). San Diego, CA: Academic Press.

Cannon, W. B. (1932 ). The wisdom of the body. The American Journal of the Medical Sciences, 184, 864. doi: http://dx.doi.org/10.1097/00000441- 193212000-00028

Castonguay, L. G. Muran, J. C. (2015). Fostering collaboration between researchers and clinicians through building practice-oriented research: an introduction. Psychotherapy Research, 25(1), 1-5. doi:10.1080/ 10503307.2014 .966348

Cristea, I. A., Gentili, C., Cotet, C. D., Palomba, D., Barbui, C. y Cuijpers, P. (2017). Efficacy of Psychotherapies for Borderline Personality Disorder: A Systematic Review and Meta-analysis. JAMA Psychiatry, 74(4), 319-328. doi:10.1001/jamapsychiatry.2016.4287

Cuijpers, P., Karyotaki, E., Reijnders, M. Ebert, D. D. (2018). Was Eysenck right after all? A reassessment of the effects of psychotherapy for adult depression. Epidemiology and Psychiatric Sciences, 1-10. doi:10.1017/ S2045796018000057

Diaz-Garcia, A., Gonzalez-Robles, A., Fernandez-Alvarez, J., Garcia-Palacios, A., Banos, R. M. y Botella, C. (2017). Efficacy of a Transdiagnostic internet-based treatment for emotional disorders with a specific component to address positive affect: Study protocol for a randomized controlled trial. BMC Psychiatry, 17, 145. doi:10.1186/s12888-017-1297-z

Dixon-Gordon, K. L., Bernecker, S. L. y Christensen, K. (2015). Recent innovations in the field of interpersonal emotion regulation. Current Opinion in Psychology, 3, 36-42. doi:10.1016/j.copsyc.2015.02.001

Extremera, N. y Rey, L. (2015). The moderator role of emotion regulation ability in the link between stress and wellbeing. Frontiers in Psychology, 6, 1632. doi:10.3389/fpsyg.2015.01632

Fernández-Álvarez, J., Fernández-Álvarez, H. y Castonguay, L. (2018). Summarizing novel efforts to integrate practice and research from a practice oriented research perspective. Revista Argentina de Clínica Psicológica, 27(2), 363-372.

Gaggioli, A., Pallavicini, F., Morganti, L., Serino, S., Scaratti, C., Briguglio, M., Crifaci, G., Vetrano, N., Giulintano, A., Bernava, G., Cipresso, P., Vigna, C., Grassi, A., Baruffi, M., Wiederhold, B. y Riva, G. (2014). Experiential virtual scenarios with real-time monitoring (interreality) for the management of psychological stress: a block randomized controlled trial. Journal of Medical Internet Research, 16(7), e167. doi:10.2196/jmir.3235

Gonzalez-Blanch, C. s., Umaran-Alfagemea, O., Cordero-Andres, P., Muñoz-Navarro, R., Ruiz-Rodriguez, P., Medrano, L. A., Ruiz-Toress, M., Collado, E. D. y Cano-Vindel, A. (2018). Tratamiento psicologico de los trastornos emocionales en Atencion Primaria: el manual de tratamiento transdiagnostico del estudio PsicAP. Ansiedad y Estrés, 24(1), 1-11. doi:10.1016/j.anyes.2017.10.005

González-Robles, A., García-Palacios, A., Baños, M. R., Riera, A., Llorca, G., Traver, F., Haro, G., Palop, V., Lera, G., Romeu, J. E. y Botella, C. (2015). Effectiveness of a transdiagnostic internet- based protocol for the treatment of emotional disorders versus treatment as usual in specialized care: study protocol for a randomized controlled trial. Trials, 16. doi:10.1186/s13063-015-1024-3

Gratz, K. L. y Roemer, L. (2004). Multidimensional Assessment of Emotion Regulation and Dysregulation: Development, Factor Structure, and Initial Validation of the Difficulties in Emotion Regulation Scale. Journal of Psychopathology and Behavioral Assessment, Vol. 26(1), 41-54.

Grill, S. S., Castañeiras, C. y Fasciglione, M. P. (2017). Aplicacion grupal del Protocolo Unificado para el tratamiento transdiagnostico de los trastornos emocionales en poblacion argentina. Revista de Psicopatología y Psicología Clínica, 22(3), 171-181. doi:10.5944/rppc.vol.22.num.3.2017.18122

Gross, J. J. (1998). The Emerging Field of Emotion Regulation: An Integrative Review. Review of General Psychology, 2(3), 271-299. 
Gross, J. J. (2015a). Emotion Regulation: Current Status and Future Prospects. Psychological Inquiry, 26(1), 1-26. doi:10.1080/1047840X.2014.940781

Gross, J. J. (2015b). Handbook of Emotion Regulation (Second Edition). Nueva York, NY: The Guilford Press.

Guillen, V., Garcia-Palacios, A. y Banos, R. (2018). Terapia dialectica comportamental para un paciente con trastorno bipolar I: un estudio de caso con seguimiento a los dos anos. Revista Argentina de Clínica Psicológica, 27(1), 14-35. doi:10.24205/03276716.2018.1045

Gyani, A., Shafran, R., Myles, P. y Rose, S. (2014). The gap between science and practice: how therapists make their clinical decisions. Behavior Therapy, 45(2), 199-211. doi:10.1016/j.beth.2013.10.004

Harvey, A. G., Watkins, E., Mansell, W. y Shafran, R. (2004). Cognitive Behavioural Processes Across Psychological Disorders: A Transdiagnostic Approach to Research and Treatment. Oxford, Reino Unido: Oxford University Press.

Hershenberg, R. y Goldfried, M. R. (2015). Implications of RDoC for the Research and Practice of Psychotherapy. Behavior Therapy, 46, 156-165.

Hofmann, S. G. (2014). Interpersonal Emotion Regulation Model of Mood and Anxiety Disorders. Cognitive Therapy and Research, 38(5), 483-492. doi:10.1007/s10608-014-9620-1

Hofmann, S. G. y Asmundson, G. J. (2008). Acceptance and mindfulness-based therapy: new wave or old hat? Clinical Psychology Review, 28(1), 1-16. doi:10.1016/j.cpr.2007.09.003

Hofmann, S. G. y Hayes, S. C. (2018). The Future of Intervention Science: Process-Based Therapy. Clinical Psychological Science, 2167702618772296. doi:10.1177/2167702618772296

Insel, T., Cuthbert, B., Garvey, M., Heinssen, R., Pine, D. S., Quinn, K., Sqanislow, C. y Wang, P. (2010). Research Domain Criteria (RDoC): Toward a New Classication Framework for Research on Mental Disorders. American Journal of Psychiatry, 167(7), 748-751.

Kanfer, F. H. y Hagerman, S. (1987). A Model of Self-Regulation. Berlin, Alemania: Heidelberg.

Kirwan, M., Pickett, S. M. y Jarrett, N. L. (2017). Emotion regulation as a moderator between anxiety symptoms and insomnia symptom severity. Psychiatry Research, 254, 40-47. doi:10.1016/j.psychres.2017.04.028

Koole, S. L. y Veenstra, L. (2015). Does Emotion Regulation Occur Only Inside People's Heads? Toward a Situated Cognition Analysis of Emotion-Regulatory Dynamics. Psychological Inquiry, 26(1), 61-68. doi:10.1080/ 1047840X.2015.964657

Kotov, R., Krueger, R. F., Watson, D., Achenbach, T. M., Althoff, R. R., Bagby, R. M., Brown, T. A., Carpenter, W. T., Caspi, A., Clark, L. A., Eaton, N. R., Forbes, M. K., Forbush, K. T., Goldberg, D., Hasi, D., Hyman, S. E., Ivanova, M. Y., Lynam, D. R., Markon, K., Miller, J. D., Moffitt, T. E., Morey, L. C., Mullins-Sweatt, S. N., Ormel, J., Patrick, C. J., Regier, D. A., Rescorta, L., Ruggero, C. J., Samuel, D. B., Sellbom, M., Simms, L. J., Skodol, A. E., Slade, T., South, S. C., Tackett, J. L., Waldman, I. D., Waszczuk M. A., Widiger, T. A., Wright, A. G. C. y Zimmerman, M. (2017). The Hierarchical Taxonomy of Psychopathology (HiTOP): A dimensional alternative to traditional nosologies. Journal of Abnormal Psychology, 126(4), 454-477. doi:10.1037/abn0000258

Krueger, R. F. y Markon, K. E. (2006). Reinterpreting Comorbidity: A Model-Based Approach to Understanding and Classifying Psychopathology. Annual Review of Clinical Psychology, 2, 111-133.

LeDoux, J. E. y Hofmann, S. G. (2017). The subjective experience of emotion: a fearful view. Current Opinion in Behavioral Sciences, 19, 67-72. doi:10.1016/j.cobeha.2017.09.011

Loo Gee, B., Griffiths, K. M. y Gulliver, A. (2016). Effectiveness of mobile technologies delivering Ecological Momentary Interventions for stress and anxiety: a systematic review. Journal of the American Medical Informatics Association, 23(1), 221-229. doi:10.1093/jamia/ocv043

Mahaffey, B. L., Watson, D., Clark, L. A. y Kotov, R. (2016). Clinical and personality traits in emotional disorders: Evidence of a common framework. J Abnorm Psychol, 125(6), 758-767. doi:10.1037/abn0000187

Malik, S., Wells, A. y Wittkowski, A. (2015). Emotion regulation as a mediator in the relationship between attachment and depressive symptomatology: A systematic review. Journal of Affective Disorders, 172, 428444. doi:10.1016/j.jad.2014.10.007

Mansell, W. y Marken, R. S. (2015). The Origins and Future of Control Theory in Psychology. Review of General Psychology, 19(4), 425-430. doi:10.1037/gpr0000057

Miller, G. (2012). The Smartphone Psychology Manifesto. Perspectives on Psychological Science, 7(3), 221-237.

Neacsiu, A. D., Bohus, M. y Linehan, M. (2014). Dialectical behavior therapy: An intervention for emotion dysregulation. En J. J. Gross (Ed.), Handbook of emotion regulation (pp. 491-507). Nueva York, NY: Guilford Press.

Osbeck, L. M. (2009). Transformations in Cognitive Science: Implications and Issues Posed. Journal of Theoretical and Philosophical Psychology, 29(1), 16-33. doi:10.1037/a0015454 
Osma, J., Suso-Ribera, C., Garcia-Palacios, A., Crespo-Delgado, E., Robert-Flor, C., Sanchez-Guerrero, A., Ferreres-Galan, V., Pérez-Ayerra, L., Malea-Fernández, A. y Torres-Alfosea, M. A. (2018). Efficacy of the unified protocol for the treatment of emotional disorders in the Spanish public mental health system using a group format: study protocol for a multicenter, randomized, non-inferiority controlled trial. Health and Quality of Life Outcomes, 16(1), 46. doi:10.1186/s12955-018-0866-2

Patten, S. B., Williams, J. V. A., Lavorato, D. H., Bulloch, A. G. M., Wiens, K. y Wang, J. (2016). Why is major depression prevalence not changing? Journal of Affective Disorders, 190, 93-97. doi:10.1016/ j.jad.2015.09.002

Perna, G., Grassi, M., Caldirola, D. y Nemeroff, C. B. (2018). The revolution of personalized psychiatry: will technology make it happen sooner? Psychological Medicine, 48(5), 705-713. doi:10.1017/ S0033291717002859

Porges, S. W. (2007). The Polyvagal Perspective. Biological Psychology, 74(2), 116-143.

Riva, G., Raspelli, S., Algeri, D., Pallavicini, F., Gorini, A., Wiederhold, B. K. y Gaggioli, A. (2010). Interreality in Practice: Bridging Virtual and Real Worlds in the treatment of Posttraumatic Stress Disorders. Cyberpsychology, Behavior \& Social Networking, 13 (1), 55-65. doi:10.1089/cyber.2009.0320

Sandín, B., Chorot, P. y Valiente, R. M. (2012). Transdiagnóstico: nueva frontera en psicología clínica Revista de Psicopatología y Psicología Clínica, 17(1), 185-203.

Shapero, B. G., Abramson, L. Y. y Alloy, L. B. (2016). Emotional Reactivity and Internalizing Symptoms: Moderating Role of Emotion Regulation. Cognitive Therapy and Research, 40(30), 328-340. doi:10.1007/ s10608-015-9722-4

Sloan, E., Halla, K., Mouldinga, R., Brycec, S., Mildreda, H. y Staigerb, P. K. (2017). Emotion regulation as a transdiagnostic treatment construct across anxiety, depression, substance, eating and borderline personality disorders: A systematic review. Clinical Psychology Review, 57, 141-163. doi:10.1016/j.cpr.2017.09.002

Stanton, K., Rozek, D. C., Stasik-O’Brien, S. M., Ellickson-Larew, S. y Watson, D. (2016). A transdiagnostic approach to examining the incremental predictive power of emotion regulation and basic personality dimensions. Journal of Abnormal Psychology, 125(7), 960-975. doi:10.1037/abn0000208

Strauss, G. P., Ossenfort, K. L. y Whearty, K. M. (2016). Reappraisal and Distraction Emotion Regulation Strategies Are Associated with Distinct Patterns of Visual Attention and Differing Levels of Cognitive Demand. Plos One, 11(11), e0162290. doi:10.1371/journal.pone.0162290

Thayer, J. F. y Lane, R. D. (2009). Claude Bernard and the heart-brain connection: further elaboration of a model of neurovisceral integration. Neurosci Biobehav Rev, 33(2), 81-88. doi:10.1016/j.neubiorev.2008.08.004

Tull, M. T., Bardeen, J. R., DiLillo, D., Messman-Moore, T. y Gratz, K. L. (2015). A prospective investigation of emotion dysregulation as a moderator of the relation between posttraumatic stress symptoms and substance use severity. Journal of Anxiety Disorders, 29, 52-60. doi:10.1016/j.janxdis.2014.11.003

Tyron, W. W. (2018). Mediators and Mechanisms. Clinical Psychological Science. doi:10.1177/2167702618765791

Vohs, K. D. y Baumeister, R. F. (2016). Handbook of Self-Regulation: Research, Theory, and Applications. Nueva York, NY: Guilford Press.

Von Bertalanffy, L. (1967). General theory of systems : Application to psychology. Information (International Social Science Council), 6(6), 125-136. doi:10.1177/053901846700600610

Webb, T. L., Miles, E. y Sheeran, P. (2012). Dealing with feeling: a meta-analysis of the effectiveness of strategies derived from the process model of emotion regulation. Psychological Bulletin, 138(4), 775-808. doi:10.1037/ a0027600

WHO. (2017). Depression and Other Common Mental Disorders Global Health Estimates.

Williams, J. M. G. (2007). The mindful way through depression: Freeing yourselffrom chronic unhappiness. Nueva York, NY: Guilford Press.

Wolitzky-Taylor, K. B., Horowitz, J. D., Powers, M. B. y Telch, M. J. (2008). Psychological approaches in the treatment of specific phobias: a meta-analysis. Clinical Psychology Review, 28(6), 1021-1037. doi:10.1016/ j.cpr.2008.02.007

Zaki, J. y Williams, W. C. (2013). Interpersonal emotion regulation. Emotion, 13(5), 803-810. doi:10.1037/ a0033839 